\title{
The Future of Brain-Computer Interfacing (keynote paper)
}

\author{
Anton Nijholt \\ University of Twente, Enschede, the Netherlands \\ Imagineering Institute, Iskandar, Johor Bahru, Malaysia \\ anijholt@cs.utwent.nl
}

\begin{abstract}
In this paper we survey some early applications and research on brain-computer interfacing. We emphasize and revalue the role the views on artistic and playful applications have played. In previous years various road maps for BCI research appeared. The interest in medical applications has guided BCI research, has led to necessary and useful research results, but also has led to some narrow-mindedness of choosing challenges in research. New challenges are now coming from applications that were already proposed decades ago, but didn't get attention because they focused on playfulness and 'regular' users rather than on efficiency of applications and disabled users.
\end{abstract}

Keywords-Brain-Computer Interfaces (BCI); BCI road maps; application scenarios; passive BCI; human-computer interaction; multimodal interaction; entertainment and artistic BCI applications

\section{INTRODUCTION}

In the last decade brain-computer interfacing (BCI) research has emerged from clinical applications (the treatment of patients) and has now become a theme in human-computer interaction (HCI) research in general, in multimodal interaction research, in affective computing, and in entertainment and game research. There are many reasons and causes that can be mentioned to explain the growth of interest among researchers in these areas and, partly but not unimportantly, also as a consequence of this research interest, interest of funding agencies, public media, and other users than patients with physical and mental disabilities. In the next sections of this short paper we will first give a short survey of how BCI has been used for non-clinical applications, in particular in entertainment and artistic applications.

These applications paved the way for non-clinical BCI applications in the current and next decades. Moreover, these applications make clear that BCI researchers or rather HCI researchers need to be interested in making brain activity part of the whole of modalities that can be integrated and can play a role in interpreting a user's activity, preferences and interactions with the environment. It should be mentioned that this will not necessarily require the translation of brain activity to explicit user's commands. Brain activity can be monitored just as we can monitor physiological information in general or nonverbal behavior and use this information to better interpret a user's command, get knowledge about a user's preferences and maybe change the conditions for interaction and make changes to the environment that allow smoother interaction. We will illustrate these observations on BCI research with some road mapping activities in Europe and other countries, and will add our own observations on future developments in BCI research.

\section{EARLY INTEREST AND DEVELOPMENTS IN BCI RESEARCH}

Early interest in BCI didn't always address approaches to BCI that aimed at developing knowledge about the brain and how to use this knowledge in applications. Research and influential papers that created the BCI research area are the 1924 experiments of Hans Berger on the measuring of electrical activity (alpha waves) in the human brain. Various other discoveries should be mentioned, such as the discovery of different frequencies, stimuli, disturbances, and how brain activity relates to the different regions in the brain. Kamiya [1] in 1968 made clear that humans can purposely manipulate their brain activity - he considered alpha waves - and that such changes in brain activity can be measured (Fig. 1).

At that time artists also discovered how such activity could be used in the generation and manipulation of visual representation of brain activity of one or more subjects. With two or more subjects there can be interaction and the subjects being aware, because of the (audio-)visual feedback they receive, of each other's brain activity.

\section{Conscious control of brain waves.}

Indicates the possibility of teaching "man to perceive and control some of his brain functions." After Ss had learned to distinguish between alpha and nonalpha states, they were able to consciously produce the alpha state. Control of alpha rhythms was monitored by EEGs. Experienced Zen mediators "learned control of their alpha waves far more rapidly than did the average person ... The possible value in studies of alpha wave control during the LSD experience is indicated. The lack of connection between alpha waves and extrasensory perception is stressed. "No evidence of electromagnetic radiation to the outside world by brain activity ..." was found. Studies with groups other than college educated Ss are suggested.

Figure 1. Abstract of Joe Kamiya's 1968 paper on conscious control of brain waves. 


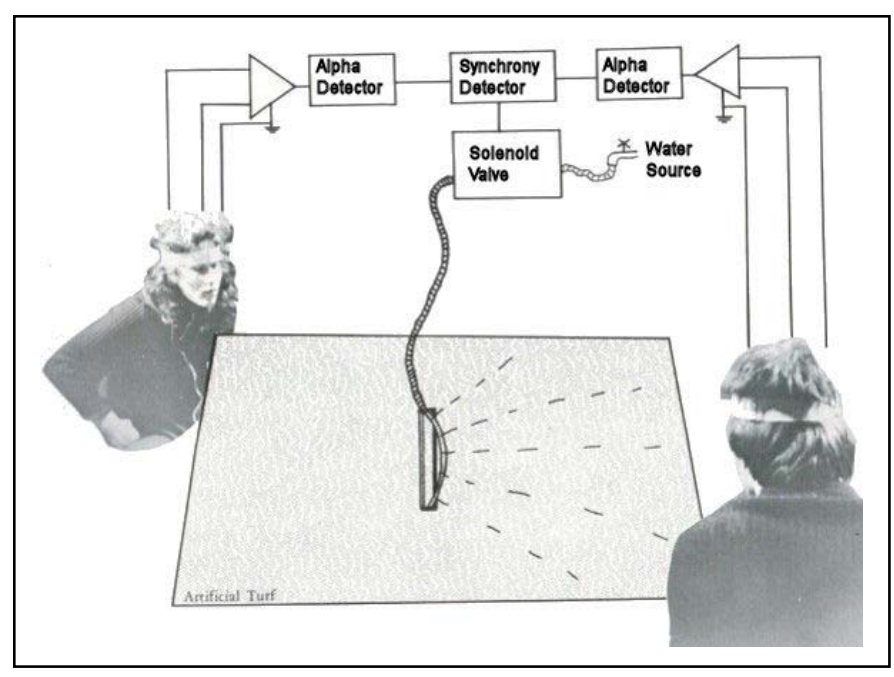

Figure 2. A synchrony detector triggers the opening of a solenoid valve, thereby controlling the flow of water through a garden hose and lawn sprinkler system.

Hence, artists embraced the idea of using brain signals to design and create artistic, playful, and interactive installations. Many of these ideas are collected in [2]. Among them is for example 'Alpha Garden' by Jacqueline Humbert in 1973, who sketched a situation where two persons control the flow of water through a garden hose and sprinkler system by synchronizing their alpha activity (Fig. 2). As another example she designed 'Brainwave Etch-a-Sketch' where two participants control horizontal and vertical movements of a dot on a screen in order to make drawings. In addition we should mention the artist Nina Sobell [3], who in the early 1970s asked participants in her experiments to synchronize their brain activity in order to achieve a joint result (an attractive visualization of their joint brain activity). In these early years the artists' use of brain activity in order to explore new ways of creating playful interactive art often assumed more than one active participant and, in addition, also often assumed an audience, turning the use of brain activity into a musical or audio-visual media performance.

There are also examples of artists who designed BCI experiments and used BCI for artistic expression during the decades between 1980 and now. However, this interest didn't really lead to new developments. The same ideas were used over and over again, but now using new media and interaction technology, rather than using new ideas generated from advances in measuring, identifying and evoking brain activity. These advances started with a paper by Jacques Vidal in 1973 [4] a paper that now is considered to be the start of BCI research. Vidal asked the now famous question: "Can these observable electrical brain signals be put to work as carriers of information in man-machine communication or for the purpose of controlling such external apparatus as prosthetic devices or spaceships?" And, he added: "Even on the sole basis of the present states of the art of computer science and neurophysiology, one may suggest that such a feat is potentially around the corner."

Vidal drew attention to 'evoked responses' of the brain, embedded in ongoing electrical activity, because of sensory stimuli (visual, auditory, somesthetic). Embedded in many technical details concerning EEG signal analysis, artefact correction, and computer architecture, we can find suggestions about how to use his approach in interactive man-computer communication "... such as: recognition of a clue (or matching), its acceptance and rejection, choice between (visual) alternatives, arbitrary positioning of a pointer on a screen, etc.". Some planned experiments are discussed to distinguish between voluntary and subconsiously evoked responses. For the latter experiments a space war game is proposed where gamers (one of them is the computer) can fire missiles at opponent's space ships. What difference will be measured in evoked potentials, given a subject's different mental state, by the explosion of either the subject's space ship or that of its opponent? His choice of examples (cursor control, games) is remarkable at a time when there were no personal computers at all. However, in computer science research, universities and research labs, these topics, but not yet the BCI approach, were well recognized.

\section{BCI AND MEDICAL APPLICATIONS}

Suggestions that user- or environment-evoked changes in brain activity can be detected and can be translated into commands, turned out to be eye openers for researchers whose interest was in assistive technologies for disabled persons, persons in the process of rehabilitation after an accident or a stroke, and patients suffering from loss of muscle control. For that reason, after the $1970 \mathrm{~s}$, interest and funded BCI research focused on clinical and medical BCI research, that is, research devoted to providing disabled persons with possibilities to communicate with their environment, including both sending verbal messages and non-verbal physical activity requiring BCI control of artificial limbs. This interest was fed by obtaining knowledge about the various BCI paradigms or BCI markers, such as event related desynchronization (ERD) and motor imagery, event related potentials (ERP), stimulation (evoked potentials) and detectable changes in brain activity patterns related to levels of relaxation. These BCI paradigms have led to many BCI applications in the medical domain. For example, grasping a glass with a prosthetic arm, composing a verbal message, or controlling the movements of a wheelchair and navigating in a physical or virtual environment. Obviously, during the early years of BCI research, apart from yet to develop knowledge about signal processing, pattern recognition, and machine learning, there were no simple ways, as we can do now with audio-visual media, to provide BCI users with stimuli from the environment, feedback from the environment and control of devices in the environment.

\section{BCI STATE OF THE ART R\&D}

From an application point of view, at this moment we can say that nowadays BCI research addresses the application scenarios that are identified in Fig. 3. This figure has been introduced in the EU funded research project BNCI Horizon 2020 meant to provide a vision on fruitful and to be funded BCI research between now and 2020 and beyond [5,6]. It should be mentioned that other road maps were available at the time the BNCI Horizon 2020 road map was developed $[7,8]$. 


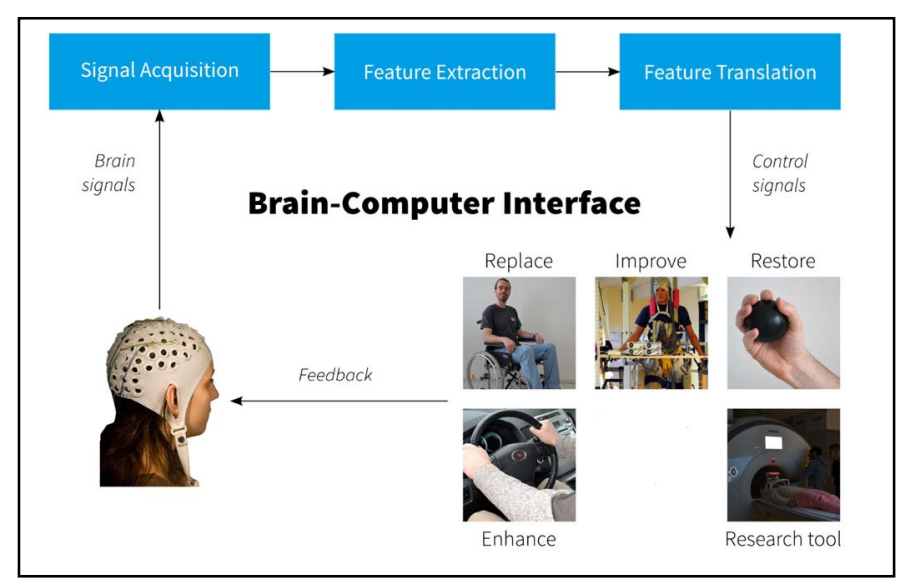

Figure 3. Application Scenarios (BNCI Horizon 2020 road map [5]).

In the BNCI Horizon 2020 road map five application scenarios are distinguished: (1) BCIs can replace functions that were lost due to injury or disease (for example, communication and wheelchair control); (2) BCIs can be used to improve functions (for example, in stroke rehabilitation); (3) BCIs can restore lost functions (for example, stimulation of muscles in a paralyzed person and stimulation of nerves to restore bladder function); (4) BCIs can enhance functions (for example, detection of stress levels or lapses of attention during demanding tasks); (5) BCIs can also be used as a research tool to study brain functions.

It is a little embarrassing, although this is slightly repaired in the appendices of this road map, how biased the description of these scenarios is against non-medical applications, such as applications in games, entertainment and arts and in humancomputer interaction in general. BCI research in the context of games and entertainment has introduced many new challenges, opening new roads and new research approaches, not necessarily aiming at efficiency and robustness, but taking more importance in fun, experience, and social aspects [9,10,11]. Moreover, since we are talking about non-disabled users, from the beginning this research has addressed the issue of multimodal interaction and embedding BCI control opportunities in a variety of interaction modalities. BCI research as part of human-computer interaction, in particular affective human-computer interaction, is also addressing the monitoring of a user's affective state in order to use such information to adapt the environment to a particular user, whether he or she is performing an office task, is active in a domestic environment, or is not active at all, for example sleeping. This can also include reducing or increasing the task complexity, for example in games or educational applications, to increase efforts to keep the user focused on his or her task, or to increase efforts that keep the user in a relaxed state. We will return to these observations in a next section, but here we conclude that such issues are hardly mentioned in the main document resulting from the BNCI Horizon 2020 project.

The application scenarios we mentioned above can be matched with technology sectors. In Table I we display the results of this matching as it appeared in an Appendix [12] of our BNCI Horizon 2020 Road Map study. The 'replace' scenario is usually about replacing a natural output that has been lost as a result of an injury or disease. BCI can be used for wheelchair control, in-house light control, bed position control, et cetera. In addition it can allow 'locked-in' patients with the possibility to communicate with others. There will be impact on the technology sector (accessories, communication \& control) of industry. 'Restore' addresses lost natural output and attempts to restore it, for example by muscle stimulation, where 'improve' aims at improving natural central nervous system output (for example, improve functions that have been impaired. Applications may have impact on the medtech, rehabilitation and robotics sectors of industry. The 'enhance' application scenario, to be found in most market application groups, is about 'enhancing' natural central nervous system output. Here we can find BCIs to monitor attention level, to adapt interfaces to a mental or affective state of a user and to enhance experience in art and gaming.

\section{TABLE I. BCI MARKET APPLICATIONS}

\begin{tabular}{|c|c|c|}
\hline $\begin{array}{l}\text { Application } \\
\text { scenario }\end{array}$ & $\begin{array}{c}\text { Market } \\
\text { application } \\
\text { groups } \\
\end{array}$ & $\begin{array}{c}\text { Key BCI-related market } \\
\text { applications }\end{array}$ \\
\hline $\begin{array}{l}\text { replace/ } \\
\text { enhance }\end{array}$ & $\begin{array}{l}\text { Communication } \\
\text { \& Control }\end{array}$ & $\begin{array}{l}\text { Affective computing, } \\
\text { interface to smartphones, } \\
\text { multimodal interaction, } \\
\text { apparel and accessories } \\
\text { (technology sector) }\end{array}$ \\
\hline $\begin{array}{l}\text { restorel } \\
\text { improvel } \\
\text { enhance }\end{array}$ & $\begin{array}{c}\text { Health \& } \\
\text { Neurofeedback }\end{array}$ & $\begin{array}{l}\text { Prevention, diagnosis, } \\
\text { therapy, monitoring, } \\
\text { cognitive and motor } \\
\text { rehabilitation, addiction } \\
\text { disorders, wellness, } \\
\text { nutrition (medtech \& rehab } \\
\text { \& robotics sector) }\end{array}$ \\
\hline $\begin{array}{l}\text { replacel } \\
\text { enhance }\end{array}$ & $\begin{array}{c}\text { Assistive } \\
\text { Technologies \& } \\
\text { Smart Home } \\
\text { Control }\end{array}$ & $\begin{array}{l}\text { Ambience intelligence, } \\
\text { domotics, elderly care, } \\
\text { geriatric hospices } \\
\text { (technology sector) }\end{array}$ \\
\hline enhance & $\begin{array}{l}\text { Safety \& } \\
\text { Security }\end{array}$ & $\begin{array}{c}\text { Public transport } \\
\text { (automotive and aerospace } \\
\text { sectors), fire brigade, } \\
\text { police, process controls, } \\
\text { banking security, } \\
\text { agriculture }\end{array}$ \\
\hline enhance & $\begin{array}{l}\text { Entertainment } \\
\text { \& Gaming }\end{array}$ & $\begin{array}{l}\text { Educational games, serious } \\
\text { games, cinema, art, sports, } \\
\text { meditation techniques (e.g. } \\
\text { yoga, tai chi) } \\
\text { (entertainment sector) }\end{array}$ \\
\hline enhance & $\begin{array}{l}\text { Neuro- } \\
\text { marketing \& } \\
\text { Finance }\end{array}$ & $\begin{array}{l}\text { Market research, decision- } \\
\text { making studies and support } \\
\text { (marketing sector), neuro- } \\
\text { economics, stockbrokers }\end{array}$ \\
\hline research & $\begin{array}{c}\text { Research \& } \\
\text { Development }\end{array}$ & $\begin{array}{l}\text { Real-time analysis, signal } \\
\text { acquisition, signal } \\
\text { processing, output devices, } \\
\text { BCI-hybrid interfaces, } \\
\text { artificial intelligence \& } \\
\text { machine learning }\end{array}$ \\
\hline
\end{tabular}


Measuring brain activity can give information about group preferences and decision making processes. Market applications of the 'enhance' scenario can be found in the earlier mentioned sectors, but also in the automotive and aerospace sectors, the entertainment sector, and the marketing sector.

\section{VISION AND DISCUSSION}

We conclude this short paper with some observations on recent developments in BCI research that link views on BCI research and applications in the 1970s with current research and views on applications beyond the medical domain. First of all, we should mention that some previous roadmaps did pay lots of attention to BCI research in the context of other than medical applications [7,8] and clearly this happened in observations on DARPA funding [13,14]. Moreover, many research projects funded by national or EU funding programs did incorporate research devoted to other than medical BCI related research issues. A good example is the Dutch BrainGain project [15], a project initiated by the Dutch government, during 2007-2012 with a funding of almost 15 million Euros. That road map projects are initiated need to be applauded. Funding agencies and governmental departments need to know about future technology developments and strengths and weaknesses of local, regional and international research communities. In the early years of the 21th century there have been many EU funded research projects on BCI research. A table with an overview of such projects can be found in [6].

We think that some of the road maps, for example the earlier mentioned BNCI Horizon 2020 road map discussed in the previous section, are very much biased towards traditional BCI research that aims at medical, serious, and 'no-fun' applications (replace functions that were lost due to injury or disease, assist in stroke rehabilitation, restore muscle activity in paralyzed persons, stress detection). There are various possibilities to explain this. Firstly, as might be obvious, mainly research groups that have already earned their spurs are asked to contribute to new road maps. This is obvious, but this might not be a good idea when advances in technology allow new research groups to enter the field. Their new research and development areas can fall outside the interest of traditional research groups. For example, it is quite a step to make a change in thinking from very careful applying BCI research in a vulnerable patient context, including ethical considerations, towards an application of BCI research in an entertainment or artistic context, where robustness and efficiency not necessarily play a very important role, and where we prefer to introduce surprise, challenges, and fun, rather than concern, predictability and enhancement of confidence.

In human-computer interaction research in general we have an 'abled' user who can control a keyboard or a wearable, can move around and use gestures or, depending on the interface and the application, use speech, touch and eye gaze, to give commands. Also, in human-computer interaction it is useful to monitor the user and to collect knowledge about the user so that the interface knows about preferences, moods, and emotions of the user, whether he or she is involved in multiple or critical tasks and whether there is cooperation or competition with others. This information can be used to predict, anticipate, guide, and interpret the interaction that a user wants to perform.

In the first 'control' situation BCI has become part of the many interaction possibilities that technology allows us. Some of them are robust, for example keyboard and mouse interaction; others are not (yet) robust, for example gesture and eye gaze control. BCI does not score high on a scale of robustness of interaction technologies. This clearly depends on the BCI paradigm that is considered, the measuring device (number of electrodes), the training, and, of course, the application. How 'real-time' does the interaction need to be? How much robustness is required by the application? Can we, as first explained in [9], turn lack of robustness in interesting or entertaining challenges for the user? Moreover, for the 'abled' user BCI can be integrated with other interaction technologies. Hence, incomplete or ambiguous information extracted from one interaction modality can be compensated by information extracted from a different interaction modality. Lack of robustness does not necessarily mean that there will not be a useful contribution to an interaction. Also in human-human interaction we have learned to live with a lack of robustness in exchanging information, including affective information. BCI control of applications needs to be investigated in the context of multimodal interaction research [10,16,17], including (synchronized and sequential) interactions requiring more than one user [11].

In the second 'monitor' situation BCI provides us with information about the affective and mental state of the user. This information can be used to fine-tune the commands of a user, but more generally, to adapt the appearance of the interface to the user, to increase or decrease efforts asked from the user, or to adapt the (combination of) interaction possibilities to the preferences of the user or the system. In recent years this has become known as 'passive BCI' [18], but, of course, in research on human-computer interaction involving (neuro-) physiological information, investigating and monitoring brain activity during task performance BCI has played this role before, in off-line (evaluating the efficiency or user-friendliness of an interface) and on-line adaption of an interface to a user $[19,20,21]$.

These latter passive BCI applications have received much attention in previous years. A domestic, entertainment or game environment needs to know about its home inhabitants, its visitors or its gamers. Measuring the affective state of an interaction partner yields important information. A videogame environment may show that it has knowledge of a gamer's affective state and because of that increases or decreases a game level's of complexity. But it can as well allow an artistic environment to change its audio-visual appearance and as a consequence provide audio-visual feedback to a user that in turn can change this user's affective state. Moreover, once receiving this feedback, a user or the audience members involved in an artistic performance can become aware of how their brain activity has impact on the artistic application and can start cooperating or competing by trying to manipulate their brain activity. Obviously, these are quite different applications of BCI research than those that aim at improving a patient's possibility to grasp a drinking glass with an arm and hand prosthesis using motor imagery. And clearly, 
physiological information that provides knowledge about an affective state can also be obtained from other, less inconvenient devices than a current BCI headset. But this might change. Anyway, knowing about the affective state of a user, abled or disabled, helps to interpret his or her intentions, activities, and commands, even including the voluntary or externally evoked commands that can be measured from brain activity [22].

Finally, trying to close the circle, we should mention that in the previous years we not only saw an emerge of interest in using BCI for games and entertainment, but also an increase of interest in BCI for artistic expression, whether it is by an individual artist or an artistic performance involving audience members [23]. One of the interesting research issues is the control type in artistic BCIs. If a piece of art is created using a $\mathrm{BCI}$, what exactly has been the role of the BCI? A BCI can be used to control the buttons and the menus of a drawing program. This may be wonderful for an artist suffering from ALS [24], it may have impact on his or her style of drawing and painting, but it is mainly about being able to click the right buttons. This can be contrasted with a situation where voluntary and involuntary changing brain activity from one or more performers is mapped on audio-visual media or integrated with an artist's audio-visual interactive installation [25].

\section{CONCLUSIONS}

In this short paper we looked at developments in BCI research and technology. We looked at ways traditional BCI researchers, working in the medical domain, deal with these developments. Their views can be contrasted with those from BCI researchers from computer science, human-computer interaction and entertainment technology who address quite different user groups. We think that BCI research should make similar transitions than the ones that took place in computer science and computer science applications. First of all, in the late 1940s and early 1950s there were discussions about the number of computers that were needed. For example, one in England, but, maybe, for political reasons also one in Scotland? But it turned out that computers meant to do scientific calculations (from ballistic missiles to atom bombs) could as well be applied for administrative tasks, for process control in industry, and for civil and other engineering problems. Apart from some visionaries such as Vannevar Bush or later Douglas Engelbart, no-one predicted general use of and access to computing facilities. But, maybe more to the point, when in the late seventies and early eighties of the previous century hobbyists started to develop their own computing devices, the traditional computer science community didn't pay any attention. That is, the computer science research community didn't recognize how its fields of research would change because of the massive public embracement of Personal Computers, followed by Internet and World Wide Web. Neither did they foresee and take seriously the interest and the economic importance of video games. Let alone the developments we have seen in social media and the use of mobile computing devices. Nevertheless, nowadays HCI researchers are fully aware of the interaction possibilities that are offered by new technology and it is certainly not the case that applications that require efficiency are considered to be more important than applications that provide users with learning experiences, challenges, communication with friends and relatives, and gaming fun. Hopefully these different communities will have more cooperation in the future and traditional BCI researchers will accept that other than medical applications will determine the way other research communities and society in general will appreciate and give directions to BCI research.

\section{REFERENCES}

[1] J. Kamiya. "Conscious control of brain waves," Psychology Today, 1(11) pp. 56-60, 1968

[2] D. Rosenboom, Ed., Biofeedback and the Arts: results of early experiments, A.R.C. Publications, Vancouver, 1976.

[3] N. Sobell, "Streaming the brain," IEEE Multimedia 9 (3), 2002, pp. 4-8.

[4] J. Vidal, "Toward direct brain-computer communication", in Annual Review of Biophysics and Bioengineering, L.J. Mullins, Ed., Annual Reviews, Inc., Palo Alto, Vol. 2, 1973, pp. 157-180.

[5] G.R. Müller-Putz et al., Roadmap: The future in brain/neural-computer interaction: Horizon 2020. Graz: Austria: University of Graz, 2015. Downloadable from: http://bnci-horizon-2020.eu/roadmap

[6] C. Brunner et al., "BNCI Horizon 2020: Towards a Roadmap for the BCI Community," Brain-Computer Interfaces, vol. 2, issue 1, pp. 1-10, 2015.

[7] T.W. Berger et al., Brain-Computer Interfaces: An International Assessment of Research and Development Trends. Netherlands: Springer, Netherlands, 2008.

[8] B. Allison et al, Future BNCI: A Roadmap for Future Directions in Brain/Neuronal Computer Interaction, Roadmap developed by the Future BNCI Project of the Information and Communication Technologies (ICT) theme of the Seventh Framework of the European Commission, 2012. Downloadable from: http://bnci-horizon2020.eu/images/bncih2020/FBNCI_Roadmap.pdf

[9] A. Nijholt, D. Plass-Oude Bos, B. Reuderink, "Turning shortcomings into challenges: Brain-computer interfaces for games," Entertainment Computing, vol. 1, issue 2, pp. 85-94, 2009.

[10] A. Nijholt, "Multimodal and Multi-Brain Computer Interfaces," in Proceedings 10th International Conference on Information, Communications and Signal Processing (ICICS 2015), IEEE Xplore, December 2-4, 2015, Singapore, in press.

[11] A. Nijholt, "Competing and collaborating brains: multi-brain computer interfacing," Chapter 12 in Brain-Computer Interfaces: Current trends and Applications, A.E. Hassanieu and A.T. Azar, Eds. Springer International Publishing Switzerland, 2015, pp. 313-335.

[12] B. Otal et al. Eds., Appendix B Industry. Appendix of BNCI Horizon 2020. The Future of Brain/Neural Computer Interaction: Horizon 2020, Graz, Austria: University of Graz, April 2015.This appendix and the corresponding main document can be downloaded from http://bncihorizon-2020.eu/

[13] B.J. Lance, S.E. Kerick, A.J. Ries, K.S. Oie, and K. McDowell, "BrainComputer Interface Technologies in the Coming Decades," Proceedings of the IEEE, Vol. 100, Issue: Special Centennial Issue, pp. 1585-1599, 2012.

[14] R.A. Miranda et al., "DARPA-funded efforts in the development of novel brain-computer interface technologies," Journal of Neuroscience Methods, Vol. 244, pp. 52-67, 2015.

[15] A. Nijholt, J. van Erp, and D.K.J. Heylen, "BrainGain: BCI for HCI and Games," in Proceedings Symposium Brain Computer Interfaces and Human Computer Interaction: A Convergence of Ideas at the AISB 2008 Convention Communication, Interaction and Social Intelligence, Aberdeen, 2 April 2008, The Society for the Study of Artificial Intelligence and Simulation of Behaviour, Brighton, UK, pp. 32-35.

[16] H. Gürkök and A. Nijholt, "Brain-computer interfaces for multimodal interaction: a survey and principles," International Journal of HumanComputer Interaction, Vol. 28, Nr. 5, pp. 292-307, 2012. 
[17] A. Nijholt, B.Z. Allison, and R.K. Jacob, "Brain-Computer Interaction: Can Multimodality Help?" in Proceedings 13th International Conference on Multimodal Interaction, H. Bourlard, T.S. Huang, E. Vidal, D. Gatica-Perez, L.-P. Morency, N. Sebe (Eds.), Alicante, Spain, 14-18 November 2011, ACM Digital Library, pp. 35-39.

[18] L. George and A. Lécuyer, "An overview of research on 'passive' braincomputer interfaces for implicit human-computer interaction," in International Conference on Applied Bionics and Biomechanics ICABB 2010-Workshop W1 'Brain-Computer Interfacing and Virtual Reality', Oct 2010, Venice, Italy.

[19] D. Chen and R. Vertegaal, "Using mental load for managing interruptions in physiologically attentive user interfaces," in CHI '04 Extended Abstracts on Human Factors in Computing Systems (CHI EA '04). ACM, New York, NY, USA, 2004, pp. 1513-1516.

[20] E. Cutrell and D. S. Tan, "BCI for passive input in HCI," in Proc. Workshop on Brain-Computer Interfaces for HCI and Games, joint with ACM CHI Conference on Human Factors in Computing Systems, 2007.
[21] A. Girouard, "Adaptive brain-computer interface," in CHI '09 Extended Abstracts on Human Factors in Computing Systems (CHI EA '09). ACM, New York, NY, USA, 2009, pp. 3097-3100.

[22] G. Garcia-Molina, T. Tsoneva, and A. Nijholt. "Emotional braincomputer interfaces," Int. J. Auton. Adapt. Commun. Syst. Vol. 6, Issue 1, pp. 9-25, 2013.

[23] H. Gürkök and A. Nijholt, "Affective Brain-Computer Interfaces for Arts," in Proc. 5th biannual Humaine Association Conference on Affective Computing and Intelligent Interaction (ACII 2013), A. Nijholt, S. D'Mello, M. Pantic (Eds.), Geneva, Switzerland, IEEE Computer Society, 2013, pp. 827-831.

[24] L. Botrel, E.M. Holz, and A. Kübler, "Brain Painting V2: evaluation of P300-based brain-computer interface for creative expression by an enduser following the user-centered design," Brain-Computer Interfaces, Vol. 2, Issue 2-3, pp. 135-149, 2015.

[25] A. Wadeson, A. Nijholt, and C.S. Nam, "Artistic brain-computer interfaces: State-of-the-Art of control mechanisms," Brain-Computer Interfaces, 2015, 2(2-3) pp. 70-75. 